\title{
Behavioral-variant frontotemporal dementia
}

\author{
Distinct phenotypes with unique functional profiles \\ OPEN
}

Claire M. O'Connor, MOT (Hons)

Ramon Landin-Romero, $\mathrm{PhD}$

Lindy Clemson, $\mathrm{PhD}$

Cassandra Kaizik, MPH

Naomi Daveson,

PostGradDipPsych

John R. Hodges, MBBS, $\mathrm{MD}$

Sharpley Hsieh, PhD

Olivier Piguet, PhD

Eneida Mioshi, PhD

Correspondence to

Prof. Mioshi:

e.mioshi@uea.ac.uk

Supplemental data at Neurology.org

\section{ABSTRACT}

Objective: To identify distinct behavioral phenotypes of behavioral-variant frontotemporal dementia (bvFTD) and to elucidate differences in functional, neuroimaging, and progression to residential care placement.

Methods: Eighty-eight patients with bvFTD were included in a cluster analysis applying levels of disinhibition and apathy (Cambridge Behavioural Inventory-Revised) to identify phenotypic subgroups. Between-group (Kruskal-Wallis, Mann-Whitney $U$ ) functional differences (Disability Assessment for Dementia) and time to residential care placement (survival analyses) were examined. Cortical thickness differences (whole-brain MRI) were analyzed in patients with bvFTD vs healthy controls ( $n=30$ ) and between phenotypic subgroups.

Results: Four phenotypic subgroups were identified: primary severe apathy ( $\mathrm{n}=26$ ), severe apathy and disinhibition ( $n=26$ ), mild apathy and disinhibition ( $n=27$ ), and primary severe disinhibition $(n=9)$. Patients with severely apathetic phenotypes were more functionally impaired and had more extensive brain atrophy than those with mild apathy or severe disinhibition alone. Further imaging analyses indicated that the right middle temporal region is critical for the development of disinhibition, an association that remains with disease progression and in the context of severe apathy. Finally, no difference in time to residential care admission was found between phenotypes.

Conclusions: This study reveals that different clinical behavioral phenotypes of bvFTD have differing profiles of functional decline and distinct patterns of associated cortical changes. These findings emphasize the importance of apathy in functional impairment, highlight the role of the right temporal region in disinhibition, and suggest that disability may be a sensitive outcome measure for treatments targeting reduction of apathy. These phenotypes could also support understanding of prognosis and clinical management. Neurology ${ }^{\circledR}$ 2017;89:570-577

\section{GLOSSARY}

ACE-III = Addenbrooke's Cognitive Examination-III; ACE-R = Addenbrooke's Cognitive Examination-Revised; $\mathbf{A D L}=$ activity of daily living; bvFTD = behavioral-variant frontotemporal dementia; $\mathbf{C B I}-\mathbf{R}=$ Cambridge Behavioural Inventory-Revised; $\mathbf{C l}=$ confidence interval; DAD = Disability Assessment for Dementia; $\mathbf{M A}+\mathbf{D}=$ mild apathy and disinhibition; $\mathbf{P S A}=$ primary severe apathy; PSD = primary severe disinhibition; $\mathbf{R O I}=$ region of interest; $\mathbf{S A}+\mathbf{D}=$ severe apathy and disinhibition.

Behavioral symptoms are salient in behavioral-variant frontotemporal dementia (bvFTD), yet considerable phenotypic variability exists within the diagnosis. ${ }^{1}$ Clinical studies have paid particular attention to the symptoms of apathy and disinhibition, which have been identified as causing the greatest caregiver distress. ${ }^{2,3}$ bvFTD has been classified into apathetic (primary symptoms of aspontaneity, inertia, and slowness) or disinhibited (more distractibility, overactivity, and restlessness) behavioral presentations. ${ }^{4}$ Subsequent studies investigated associations of these behavioral phenotypes with metabolic and neuroanatomic characteristics. ${ }^{2,3,5-10}$

\footnotetext{
From the Ageing, Work and Health Research Unit (C.M.O., L.C.), Faculty of Health Sciences, University of Sydney; Neuroscience Research Australia and University of New South Wales (R.L.-R., C.K., N.D., J.R.H., S.H., O.P.); Australian Research Council Centre of Excellence in Cognition and Its Disorders (R.L.-R., J.R.H., S.H., O.P.), University of New South Wales; Brain and Mind Centre (R.L.-R., J.R.H., S.H., O.P.), Sydney, Australia; and School of Health Sciences (E.M.), University of East Anglia, Norwich, UK.

Go to Neurology.org for full disclosures. Funding information and disclosures deemed relevant by the authors, if any, are provided at the end of the article. The Article Processing Charge was funded by the University of East Anglia.

This is an open access article distributed under the terms of the Creative Commons Attribution-NonCommercial-NoDerivatives License 4.0 (CC BY-NC-ND), which permits downloading and sharing the work provided it is properly cited. The work cannot be changed in any way or used commercially without permission from the journal.
} 
Cognitively, apathetic bvFTD phenotypes have frequently been reported as more impaired than disinhibited, ${ }^{7,11}$ but there is no consensus at present. $^{12}$ The functional implication of behavioral phenotypes on activities of daily living (ADLs) and prognosis has not been fully understood. Relative preservation of instrumental ADLs across both phenotypes has been reported, ${ }^{11}$ but instrumental ADLs have been subsequently described as more impaired in apathetic phenotypes. ${ }^{7,13}$ Indeed, increasing apathy has been correlated with declining functional ability in bvFTD. ${ }^{13,14}$

This study aimed to identify different behavioral profiles within bvFTD in a datadriven approach. We hypothesized that apathy, but not disinhibition, would be a strong contributor to functional disability; that severe apathy would be associated with a greater spread of cortical atrophy; and that severe apathy would contribute to more rapid placement to residential care.

METHODS Participants. Eighty-eight individuals diagnosed with bvFTD consecutively recruited by FRONTIER, the Frontotemporal Dementia Research Group in Sydney (Australia), were included in the study. These individuals were compared with 30 age-, sex-, and education-matched healthy controls. Patients were assessed in clinic between November 2007 and June 2015. Patients were included if they met current criteria for either possible or probable bvFTD ${ }^{1}$ (diagnoses were based on a multidisciplinary consensus, neurologist and neuropsychologist), had a reliable proxy informant to report on their behavior and everyday routine, did not have major depression or other neuropsychiatric disease, and did not have physical limitations that could affect ADLs. Disease duration was estimated at the time of diagnosis from the onset of symptoms as described by the caregiver. Controls were recruited from the Neuroscience Research Australia Volunteer database. Healthy controls scored above 88 of 100 on the Addenbrooke's Cognitive Examination-III (ACE-III) ${ }^{15}$ and 0 on the Sum of Boxes score of the Clinical Dementia Rating scale. ${ }^{16}$

Standard protocol approvals, registrations, and patient consents. This study was approved by the human ethics committees of the University of New South Wales and the South Eastern Sydney and Illawarra Area Health Service. Written consent was obtained from each participant and/or the primary caregiver.

Instruments. Behavioral symptoms were assessed with the Cambridge Behavioural Inventory-Revised (CBI-R), ${ }^{17}$ functional disability with the Disability Assessment for Dementia (DAD), ${ }^{18}$ and general cognition with the Addenbrooke's Cognitive Examination-Revised (ACE-R; used until February 2014$)^{19}$ and the ACE-III ${ }^{20}$ (used after 2014). Appendix e-1 at Neurology.org gives full details of the assessments.

Data analyses. Demographic and clinical variables. To explore the existence of clinical phenotypes within the bvFTD patient group (marked apathy or marked disinhibition on the basis of current literature), a hierarchical agglomerative cluster analysis was conducted. We applied the average-linkage method with squared euclidean distance using only the 2 behavioral subscales (disinhibition and apathy from the CBI-R) to determine the optimal number of clusters for the data. ${ }^{21}$ Analysis of the change in distance between the cluster mergers on the dendrogram generated from this analysis (figure e-1) suggested that 4 clusters were an appropriate solution for this data. ${ }^{22}$ This 4-cluster solution was validated with 2 consecutive 2-step clustering procedures using euclidean distance, each with a different information criterion (appendix e-1 gives details of the procedure). The 4 clusters were then used in the next phase of analyses to compare the bvFTD subgroups (clusters) in terms of their ADL functioning.

Demographic data across the 4 cluster groups were compared with Kruskal-Wallis tests and $\chi^{2}$ tests for sex comparisons. Kruskal-Wallis tests were also used to explore differences across clusters for each DAD area (total DAD, basic ADLs, instrumental ADLs, initiation, planning, execution). Mann-Whitney $U$ tests were then conducted for post hoc analyses with the BenjaminiHochberg procedure used for false discovery rate control of multiple comparisons. ${ }^{23}$

Finally, to understand if belonging to different bvFTD subgroup clusters would lead to more rapid placement to residential care, a Kaplan-Meier survival analysis was conducted. Placement to residential care (survival time) was defined as time from symptom onset to placement into residential care. Censoring was used for patients who were still living at home at the time of analysis or who remained at home until their death. All statistical analyses were performed with SPSS 21.0 (IBM, Armonk, NY).

Imaging data analyses. Whole-brain structural MRIs were acquired and processed for bvFTD $(\mathrm{n}=72)$ and healthy controls $(\mathrm{n}=30)$ as described in appendix e-1. Four sets of vertex-byvertex analyses were performed with general linear models to examine whole-brain differences in cortical thickness between the bvFTD subgroups resulting from the cluster analysis and healthy controls. Statistical significance was set at $p=0.001$ uncorrected for multiple comparisons. Furthermore, direct comparisons between bvFTD subgroups were carried out with $t$ test analyses to isolate the neural correlates of disease severity and disinhibition associated with the different clinical phenotypes. Statistical significance was set at $p=0.01$ uncorrected for multiple comparisons in these pairwise comparisons. A conservative cluster extent threshold of $\mathrm{k}>50 \mathrm{~mm}^{2}$ was used to minimize type I error while balancing the risk of type II error. ${ }^{24}$

Next, 4 greater cortical regions of interest (ROIs), one for each of the cortical lobes (frontal, temporal, parietal, and occipital), were selected, computed, and averaged across both hemispheres (for details, see table e-1). One-way analysis of variance was used to explore between-group differences in the average cortical thickness within these ROIs. Post hoc analyses were corrected for multiple comparisons with a Sidak adjustment. Finally, regression analyses were used to test for significant linear effects within these ROIs across the different groups. The level of significance was set at $p<0.05$.

RESULTS bvFTD phenotypes: Cluster demographics. Clusters and healthy controls were matched for age, sex distribution, and education (table 1). All cluster groups were matched for disease duration; healthy controls had better cognitive scores overall.

Are there distinct bvFTD phenotypes? Cluster solution results. Figure 1 shows the 4 patient subgroups, which were labeled to reflect clinical symptoms. Primary severe apathy (PSA; $n=26$ ) was characterized by 
Table 1 Demographics for bvFTD behavioral phenotypes (cluster groups) and healthy controls

\begin{tabular}{|c|c|c|c|c|c|c|c|c|c|c|}
\hline & \multirow[b]{2}{*}{$\begin{array}{l}\text { bvFTD all } \\
(\mathrm{n}=88)\end{array}$} & \multirow[b]{2}{*}{$\begin{array}{l}\text { PSA } \\
(n=26)\end{array}$} & \multirow[b]{2}{*}{$\begin{array}{l}S A+D \\
(n=26)\end{array}$} & \multirow[b]{2}{*}{$\begin{array}{l}M A+D \\
(n=27)\end{array}$} & \multirow[b]{2}{*}{$\begin{array}{l}\text { PSD } \\
(n=9)\end{array}$} & \multirow[b]{2}{*}{$\begin{array}{l}\text { Healthy } \\
\text { controls } \\
(\mathrm{n}=30)\end{array}$} & \multicolumn{2}{|c|}{$\begin{array}{l}\text { Across clusters and } \\
\text { healthy controls }\end{array}$} & \multicolumn{2}{|c|}{$\begin{array}{l}\text { Across clusters } \\
\text { only }\end{array}$} \\
\hline & & & & & & & $\begin{array}{l}\text { Statistic } \\
\chi\end{array}$ & $p$ & $\begin{array}{l}\text { Statistic } \\
\chi\end{array}$ & $p$ \\
\hline Age, y & $\begin{array}{l}62.15 \\
(8.68)\end{array}$ & $\begin{array}{l}63.96 \\
(7.93)\end{array}$ & $\begin{array}{l}60.00 \\
(10.52)\end{array}$ & $\begin{array}{l}62.30 \\
(7.28)\end{array}$ & $\begin{array}{l}62.67 \\
(8.87)\end{array}$ & $\begin{array}{l}64.53 \\
(4.19)\end{array}$ & 5.30 & $0.258^{a}$ & 2.52 & $0.471^{2}$ \\
\hline $\operatorname{Sex}(M / F), n$ & $54 / 34$ & $15 / 11$ & $16 / 10$ & $17 / 10$ & $6 / 3$ & $12 / 18$ & 4.42 & $0.353^{b}$ & 0.28 & $0.963^{b}$ \\
\hline Disease duration, $y$ & $\begin{array}{l}4.14 \\
(2.42)\end{array}$ & $\begin{array}{l}4.47 \\
(2.40)\end{array}$ & $\begin{array}{l}4.62 \\
(2.63)\end{array}$ & $\begin{array}{l}3.45 \\
(2.37)\end{array}$ & $\begin{array}{l}3.99 \\
(1.82)\end{array}$ & NA & NA & NA & 4.31 & $0.230^{\circ}$ \\
\hline $\begin{array}{l}\text { Baseline cognitive assessment; } \\
\text { maximum } 100 \text {, cutoff } 88 / 100\end{array}$ & $\begin{array}{l}65.54 \\
(14.83)\end{array}$ & $\begin{array}{l}61.22 \\
(14.07)\end{array}$ & $\begin{array}{l}62.59 \\
(18.25)\end{array}$ & $\begin{array}{l}70.41 \\
(9.74)\end{array}$ & $\begin{array}{l}71.50 \\
(15.00)\end{array}$ & $\begin{array}{l}95.23 \\
(3.58)\end{array}$ & 64.77 & $<0.001^{a}$ & 6.88 & $0.076^{\circ}$ \\
\hline
\end{tabular}

Abbreviations: bvFTD = behavioral-variant frontotemporal dementia; MA + D = mild apathy and disinhibition; NA = not applicable; PSA = primary severe apathy; PSD = primary severe disinhibition; SA + D = severe apathy and disinhibition.

Scores are means (SDs). Disease duration refers to the time between symptom onset and baseline assessment date.

${ }^{a}$ Kruskal-Wallis test $\mathrm{E}$.

${ }^{\mathrm{b}}$ Chi-square test.

severe apathy scores and mild disinhibition scores; severe apathy and disinhibition $(\mathrm{SA}+\mathrm{D} ; \mathrm{n}=26)$ was characterized by severe apathy and severe disinhibition scores; mild apathy and disinhibition (MA + $\mathrm{D} ; \mathrm{n}=27)$ was characterized by mild apathy and mild disinhibition; and primary severe disinhibition (PSD; $\mathrm{n}=9$ ) was characterized by mild apathy and severe disinhibition. Group differences confirmed the distinction of these 4 subgroups for both apathy $\left[\chi^{2}{ }_{(3)}=64.641, p<0.001\right]$ and disinhibition $\left[\chi^{2}{ }_{(3)}=63.122, p<0.001\right]$ CBI-R scores. Post hoc tests revealed specific cluster group differences (figure 1).

Functional disability differences across bvFTD phenotypes. Group differences were identified for every area of daily function measured: overall $\mathrm{ADLs}\left[\chi^{2}{ }_{(3)}=\right.$ 14.753, $p<0.005]$, basic ADLs $\left[\chi^{2}(3)=11.719\right.$, $p<0.01]$, instrumental ADLs $\left[\chi^{2}(3)=13.236, p<\right.$ 0.005 ], and the 3 subcomponents of activity performance: initiation $\left[\chi_{(3)}^{2}=16.068, p<0.005\right]$, planning $\left[\chi^{2}{ }_{(3)}=9.957, p<0.05\right]$, and execution $\left[\chi_{(3)}^{2}=\right.$ 10.391, $p<0.05]$. Post hoc analyses compared each cluster against the MA $+\mathrm{D}$ cluster (the least impaired phenotype). Next, the PSA and PSD clusters were compared. After multiple comparisons were controlled for, the PSA and the SA + D subgroups were more functionally impaired than the MA $+\mathrm{D}$ group for every functional area. No differences between the PSA and SA + D subgroups were found. Clinically, this translates as people who had severe

Figure 1 Levels of apathy and disinhibition across patient subgroups (clusters) identified in 2-step cluster analysis (with the Akaike criterion)

A

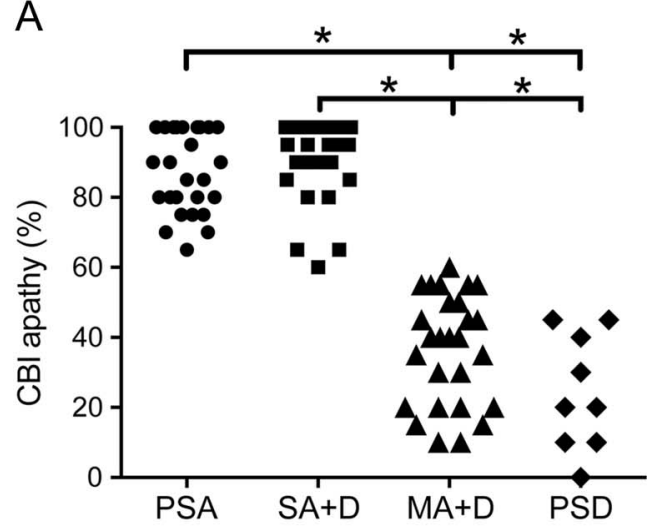

B

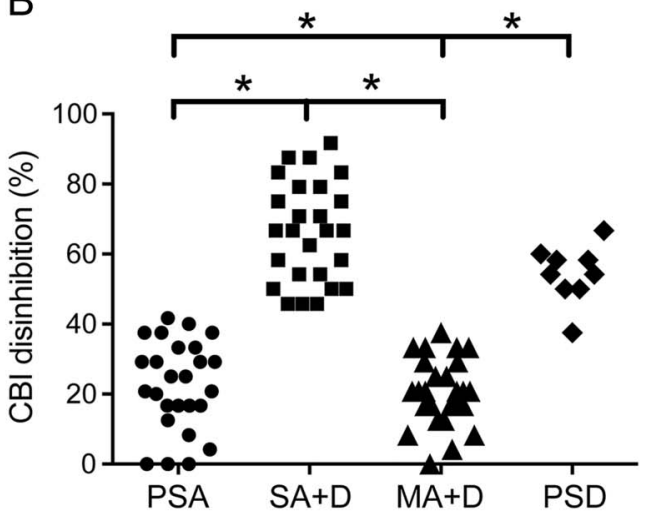

Higher scores indicate worse levels of behavioral expression. (A) PSA $>M A+D$ and PSD (both $U=0.0, p<0.001$ ), SA + D > $\mathrm{MA}+\mathrm{D}(U=0.50, p<0.001)$ and PSD $(U=0.0, p<0.001)$. (B) $S A+D>P S A$ and $M A+D($ both $U=0.0, p<0.001), P S D>$ $\mathrm{PSA}(U=3.50, p<0.001)$ and $\mathrm{MA}+\mathrm{D}(U=0.50, p<0.001)$. CBI = Cambridge Behavioural Inventory-Revised; $\mathrm{MA}+\mathrm{D}=$ mild apathy and disinhibition; PSA = primary severe apathy; $P S D$ = primary severe disinhibition; $\mathrm{SA}+\mathrm{D}=$ severe apathy and disinhibition. Mann-Whitney $U$ tests (Bonferroni correction); * $p<0.001$. 
Figure 2 Scatterplots showing scores for functional domains for each cluster phenotype

\section{A. Overall function}

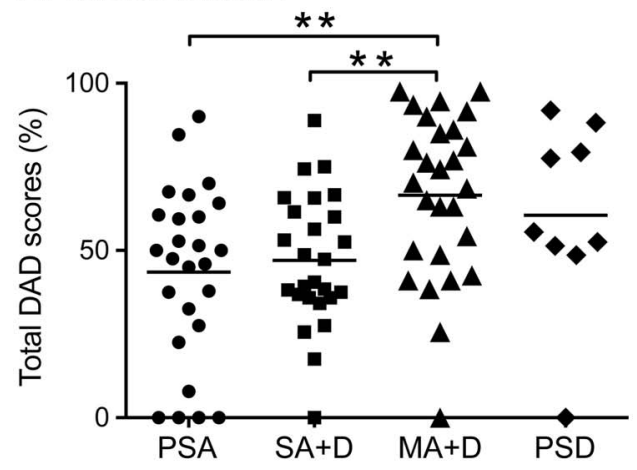

B. BADLs

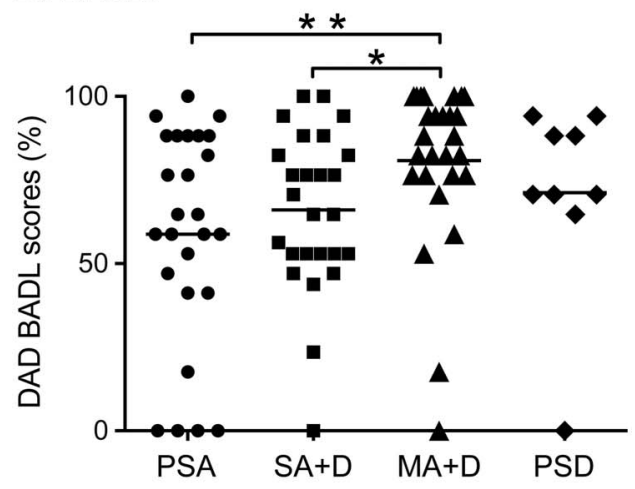

C. IADLs

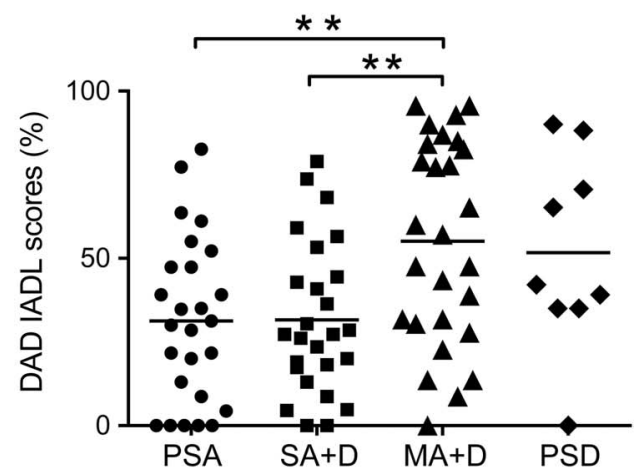

Low scores represent more impaired functional performance. $B A D L=$ basic activities of daily living; $D A D=$ Disability Assessment for Dementia; IADL = instrumental activities of daily living; MA + D = mild apathy and disinhibition; PSA = primary severe apathy; $\mathrm{PSD}=$ primary severe disinhibition; $\mathrm{SA}+\mathrm{D}=$ severe apathy and disinhibition. Mann-Whitney U tests; ${ }^{*} p<0.007$; ** $p<0.005$.

apathy were more disabled and had more difficulty with their everyday living tasks than those with mild apathy.

On the other hand, there were no differences between the PSD and MA + D groups for any of the functional domains, indicating that, in contrast to those with a primarily apathetic presentation, patients with a primarily disinhibited presentation may retain some functional abilities. Surprisingly, there were no differences between the PSA and the PSD groups. Notably, the outlier included in the PSD group $(\mathrm{n}=9)$ had a disease duration that was 5.26 years longer than the average disease duration for that group, had the equal highest concurrent levels of apathy in the PSD group, and scored zero in overall ADLs compared to the group mean of $60.57 \%$ (figure 2).

Imaging analyses. Group comparisons between bvFTD clusters and healthy controls revealed the characteristic profile of brain atrophy consistent with a diagnosis of bvFTD (figure e-2). ${ }^{8}$ In brief, all phenotypes (clusters) showed atrophy in the insula, inferior frontal, and anterior temporal cortices with some differences in the extent and location of the atrophy between subgroups. Patients in the SA + D and PSA clusters (marked apathy) exhibited an extensive and similar pattern of cortical thinning, with atrophy extending posteriorly to the temporoparietal junction and regions of the posterior cingulate cortex and precuneus. Patients in the MA $+\mathrm{D}$ cluster showed a similar pattern but with less extensive thinning. In contrast, patients in the PSD cluster (marked disinhibition) showed focal atrophy in the bilateral orbitofrontal, insular, and right anterior temporal and the anterior cingulate cortex.

Pairwise comparisons between the SA $+\mathrm{D}$ and PSA groups (both phenotypes, $\mathrm{n}=26$ ) were carried out to examine neural correlates of disinhibition, and $\mathrm{SA}+\mathrm{D}$ and $\mathrm{MA}+\mathrm{D}$ were compared to examine the neural correlates of apathy severity (figure 3). Disinhibition was associated with clusters of cortical thinning in the left temporal pole and right inferior and middle temporal gyri. Disease severity was associated with clusters of thinning in the left inferior temporal cortex and in the right insula, right middle temporal, and parietal cortices and right posterior cingulate/ isthmus.

Finally, ROI analyses showed a linear trend between symptom load/severity and cortical thinning across the different clusters (from more to less severe cortical atrophy: SA + D, PSA, MA + D, and PSD; figure 4). These analyses yielded linear associations in the frontal $\left(R^{2}=0.25, p<0.0001\right)$, temporal $\left(R^{2}=\right.$ $0.19, p<0.0001)$, parietal $\left(R^{2}=0.10, p=0.0014\right)$, and occipital $\left(R^{2}=0.10, p=0.0014\right)$ lobes. Post hoc comparisons of mean cortical thickness revealed differences between patients from all clusters and healthy controls in the frontal and temporal lobes (both $p<0.001$ ). In the parietal and occipital lobes, differences were observed between patients from the $\mathrm{SA}+\mathrm{D}$ cluster and healthy controls (both $p<0.05$ ). No differences were observed between patient subgroups. 
$S A+D$ vs $P S A$
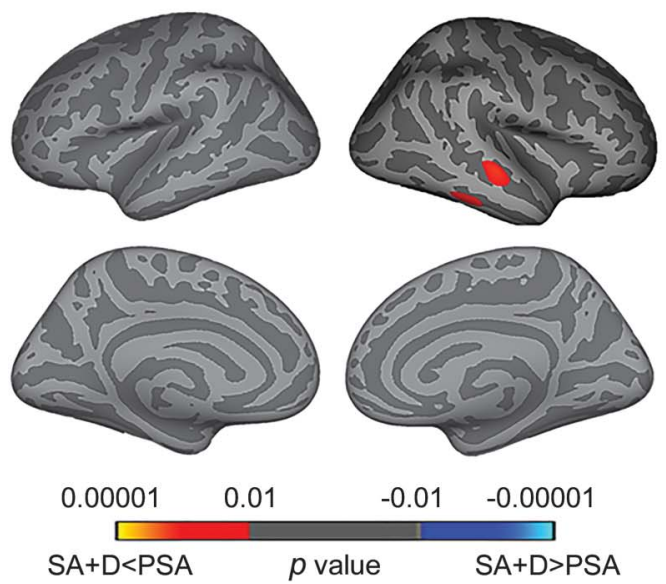

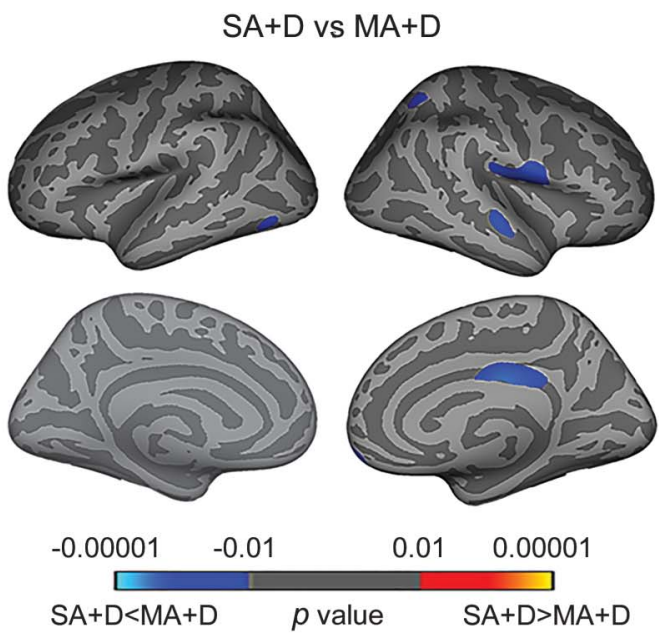

Statistical significance was set at $p=0.01$ uncorrected for multiple comparisons. bvFTD = behavioral-variant frontotemporal dementia; MA $+D=$ mild apathy and disinhibition; PSA = primary severe apathy; SA $+D=$ severe apathy and disinhibition.

bvFTD cluster phenotype and admission to residential care. At the time of the study, 38 participants had moved into residential care. One participant was excluded from the analysis because symptom onset information was unavailable. Therefore, 87 participants were included in the Kaplan-Meier survival analysis to compare the 4 patient groups in regard to admission to residential care. Overall, the median time in years from diagnosis to residential care admission for all the groups combined $(\mathrm{n}=87$ ) was 2.8 years (95\% confidence interval [CI] 2.3-3.2). The median time from diagnosis to residential care admission for each group ranged between 1.3 and 3.6 years (PSA: 1.3 years, 95\% CI 0.0-3.8; SA + D: 3.0 years, 95\% CI 1.4-4.5; MA + D: 3.1 years, 95\% CI 2.2-3.9; and PSD: 3.6 years, 95\% CI 0.4-6.9), but there were no differences between groups [figure e-3; $\left.\mathrm{X}_{(3)}=0.511, p=0.916\right]$.
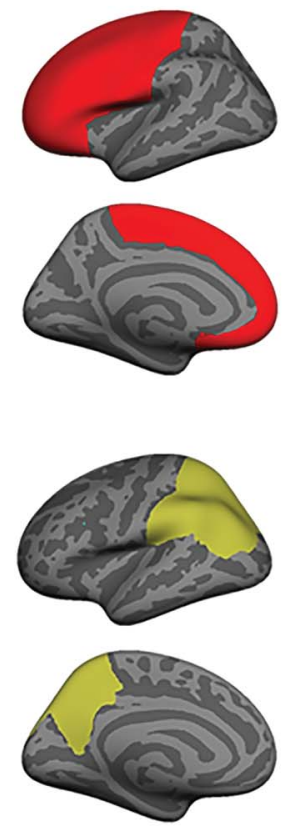

Frontal lobe
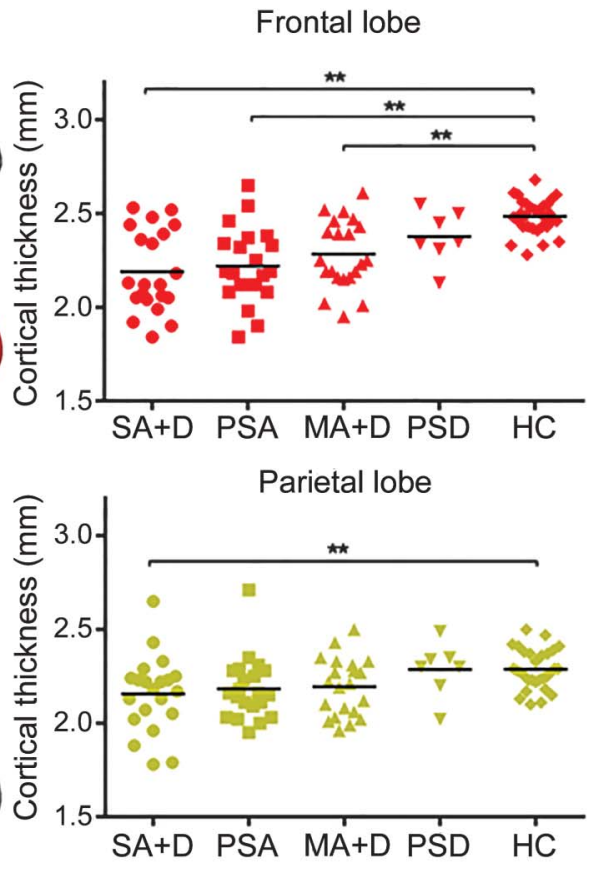
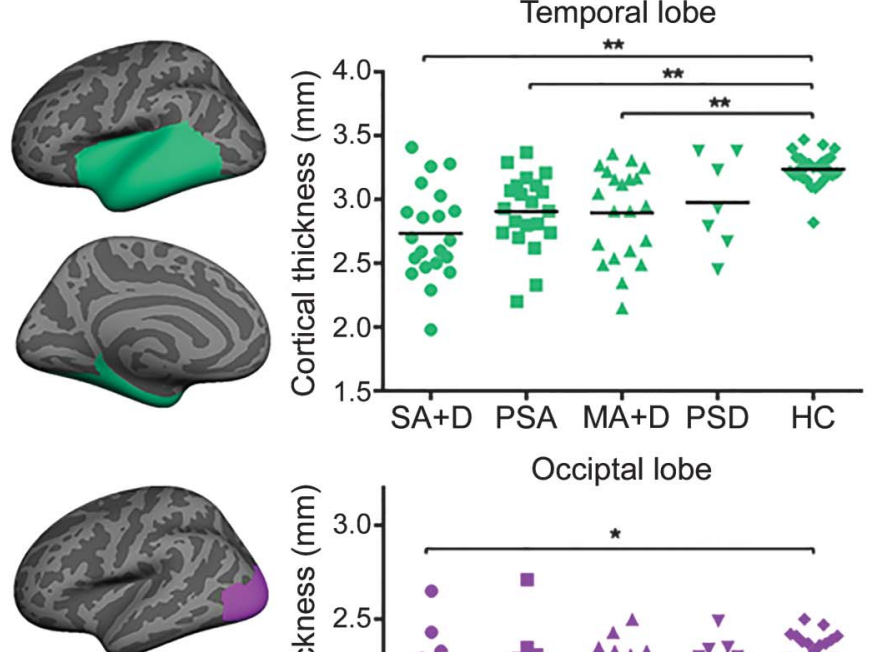

Occiptal lobe

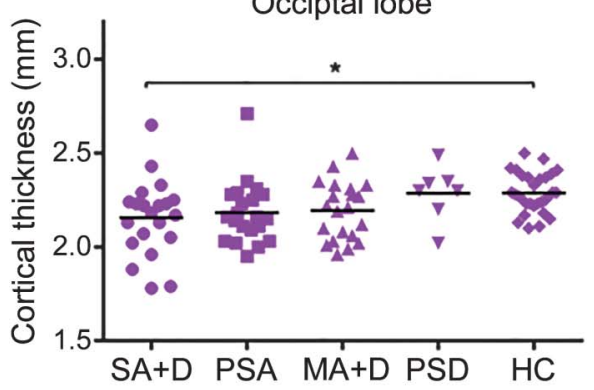

Mean cortical thickness was averaged for both hemispheres. $\mathrm{HC}=$ healthy controls; $\mathrm{MA}+\mathrm{D}=$ mild apathy and disinhibition; PSA = primary severe apathy; PSD = primary severe disinhibition; $S A+D=$ severe apathy and disinhibition. $* p<0.05 ; * *<<0.001$. 
DISCUSSION This study confirms the heterogeneity of the bvFTD syndrome on the basis of severity of apathy and disinhibited symptoms. Moreover, these different bvFTD phenotypes have been translated into distinct everyday living functional profiles and are explained by different patterns of brain atrophy. We confirmed our hypothesis that apathy, but not disinhibition, had a greater negative effect on daily functioning. Despite clear functional differences between these behavioral phenotypes, they did not appear to alter time to admission to residential care.

The recognition of different behavioral phenotypes for bvFTD is consistent with previous studies. ${ }^{8,12}$ Consensus is lacking in regard to the number of phenotypes; 2 (apathetic and disinhibited), 3 (included a mixed group), or, in the case of our study, 4 phenotypes have been reported. Similarly, 4 neuroanatomic bvFTD phenotypes have been identified in 2 separate studies; however, neither of these investigated specific apathetic or disinhibited behavioral differences. ${ }^{3,6}$ These variations are likely explained by methodologic differences such as our data-driven approach and use of different clinical assessment tools. Other reasons for the divergence in findings may include a different starting point in the analyses. Previous studies set out from neuroanatomic patterns, ${ }^{3,6}$ and some of the earlier studies predate the current bvFTD diagnostic criteria and the delineation of the C9ORF72 gene. ${ }^{1,25}$

The 4 behavioral phenotypes identified in our study did not differ in terms of disease duration, supporting the existence of distinct behavioral phenotypes beyond what could be explained by behavioral fluctuation across disease progression. Later in progression, however, the distinction becomes less clear, with increasing levels of apathy overall and any expressions of severe disinhibition likely to decline. ${ }^{8,13}$ The outlier from the PSD group provides an example of this: that patient had the longest disease duration and highest levels of apathy in the group and was much more functionally impaired.

Apathy appears to be more common than disinhibition in bvFTD. Indeed, a greater proportion of patients with severe apathy were detected in our study, reflecting previous findings. ${ }^{2,5}$ When present, disinhibition is generally accompanied by apathy. ${ }^{10,26}$ In fact, the presence of severe disinhibition without apathy was rare in our study. This profile may represent a rare phenotype, which warrants further research because it may have implications for clinical management and prognosis. Disinhibition alone did not seem to negatively influence functional impairment in bvFTD, which contrasts with previous findings. ${ }^{14}$ This inconsistency is likely to be explained by the very core definition of functional decline and selection of clinical assessments. ${ }^{16,27}$
Confirmation that severe apathy results in greater functional impairment has substantial clinical relevance for 2 reasons: apathy is pervasive in bvFTD and should be a primary focus of intervention, ${ }^{2}$ and disinhibited behaviors (e.g., shoplifting or behaving in a socially inappropriate manner) tend to dominate the clinical picture to such an extent that apathy is often overlooked. Therefore, pharmacologic and nonpharmacologic interventions to improve ADL function (or to delay disease progression because these 2 domains overlap) should emphasize addressing task initiation and planning deficits, which are commonly associated with apathy. ${ }^{28}$ Introducing activities that are intrinsically motivating and rewarding, broken down to manageable elements and incorporated into a patient's routine, has been shown to be of some benefit. ${ }^{29}$ Given the differences in functional impairment across these phenotypes, future studies should investigate the implications for caregiver burden. There is potential that caregivers may benefit from targeted approaches according to phenotype.

The 4 bvFTD phenotypes identified in our study had clearly delineated behavioral and functional differences. In contrast, no differences in general cognition were found, similar to a recent study. ${ }^{12}$ Overall, other studies demonstrated that patients with a predominantly apathetic phenotype perform more poorly on cognitive assessments than do those with a disinhibited phenotype. ${ }^{7,11}$ Variation in cognitive differences between studies may be down to the choice of general cognitive assessment tools (e.g., Mini-Mental State Examination vs ACE-R) or divergent methods of phenotypic separation (e.g., manual classification vs cluster analysis).

Disinhibited behaviors have been associated with higher levels of caregiver distress, and caregiver distress around neuropsychiatric symptoms can lead to earlier residential care admission. ${ }^{30}$ While no differences between phenotypes regarding time to residential care admission were identified in our study, information on the average time from diagnosis to residential care admission for the bvFTD cohort as a whole has clinical relevance. Our result indicating admission to residential care 2.8 years after diagnosis aligns with a recent German study that reported a median of $2.0 \pm 2.5$ years between diagnosis and residential care admission for a bvFTD cohort. ${ }^{31}$ Future studies should investigate time from diagnosis to residential care admission in a larger cohort of bvFTD phenotypes.

The 4 behavioral phenotypes identified in the cluster analysis were further supported by the imaging analyses. Compared to healthy controls, patients with the marked apathy phenotypes (PSA and SA + D) had more extensive brain atrophy than those characterized by mild apathy or marked disinhibition (MA 
$+\mathrm{D}$ and PSD). The identified differences are in line with recent studies that found that prefrontal and temporal regions were differentially associated with apathy and disinhibition in FTD. ${ }^{10,32}$ More specifically, the association with right middle temporal region in the development of disinhibition also fits with existing literature. ${ }^{10}$ This neuroanatomic delineation aligned well with the clinical group differences, which highlights that phenotypes with marked apathy are more functionally impaired than phenotypes with marked disinhibition. This, in turn, is strongly supported by our recent study that demonstrated the critical role of apathy in longitudinal functional decline in FTD. ${ }^{13}$

This study contains some limitations that need to be considered in the interpretation of these results. Foremost is the small size of the PSD subgroup, which has implications for the different group comparisons and survival and imaging analyses. Because of the small sample size of the PSD subgroup and related limited statistical power, imaging analyses were carried out uncorrected at a threshold of $p<$ 0.001 in comparisons with the control group and at $p<0.01$ in pairwise comparisons between bvFTD phenotypes. This approach, however, yielded more conservative maps of cortical differences than the standard false discovery rate correction for multiple comparisons in patients vs controls. Furthermore, these analyses were constrained with a conservative cluster extent threshold of $\mathrm{k}>50 \mathrm{~mm}^{2}$ to balance the risk of type I and type II errors. ${ }^{24}$ Finally, we did not have pathologic confirmation of our bvFTD cases. Future studies should address the differences in pathology between these behavioral phenotypes and investigate a larger cohort of the PSD phenotype. In addition, the potential link between bvFTD phenotypes and time to residential care admission needs to be further investigated.

The bvFTD phenotypic categories identified in this study could support clinical decisions on prioritization of interventions to reduce disability and to provide information on prognosis for families. Further potential for in vivo distinction of patients with differing pathologic processes could, in turn, help prevent the dilution of positive effects in therapies that occurs when patients are grouped under the single umbrella of one overarching clinical syndrome.

\section{AUTHOR CONTRIBUTIONS}

C.M. O'Connor: study design and conceptualization, data acquisition, data analysis and interpretation, statistical analysis, drafting and revision of the manuscript. R. Landin-Romero: imaging analysis and interpretation, revision of the manuscript. L. Clemson: study design and conceptualization, revision of the manuscript. C. Kaizik and N. Daveson: data acquisition, revision of the manuscript. J.R. Hodges: study supervision, revision of the manuscript. S. Hsieh: critical revision of the manuscript for intellectual content. O. Piguet: study supervision, study design and conceptualization, revision of the manuscript. E. Mioshi: study design and conceptualization, data acquisition, data analysis and interpretation, study supervision, revision of the manuscript.

\section{STUDY FUNDING}

This work was supported in part by funding to ForeFront, a collaborative research group dedicated to the study of FTD and motor neuron disease, from the National Health and Medical Research Council (APP1037746) and the Australian Research Council Centre of Excellence in Cognition and Its Disorders (CE11000102).

\section{DISCLOSURE}

C. O'Connor is a recipient of an Alzheimer's Australia Dementia Research Foundation PhD Scholarship. R. Landin-Romero is supported by the Australian Research Council Centre of Excellence in Cognition and Its Disorders Memory Node (CE11000102). L. Clemson is supported by a National Health and Medical Research Council Career Development Fellowship (APP1036639). C. Kaizik, N. Daveson, and J. Hodges report no disclosures relevant to the manuscript. S. Hsieh is supported by the Graham Linford Fellowship from the Motor Neurone Disease Research Institute of Australia. O. Piguet is supported by a National Health and Medical Research Council Senior Research Fellowship (APP1103258). E. Mioshi is supported by the Alzheimer Association USA (NIRP-12-258380) and the Alzheimer Society UK (AS-SF-14-003). Go to Neurology.org for full disclosures.

Received February 3, 2017. Accepted in final form May 12, 2017.

\section{REFERENCES}

1. Rascovsky K, Hodges JR, Knopman D, et al. Sensitivity of revised diagnostic criteria for the behavioural variant of frontotemporal dementia. Brain 2011;134:2456-2477.

2. Massimo L, Powers C, Moore P, et al. Neuroanatomy of apathy and disinhibition in frontotemporal lobar degeneration. Dement Geriatr Cogn Disord 2009;27:96-104.

3. Whitwell JL, Przybelski SA, Weigand SD, et al. Distinct anatomical subtypes of the behavioural variant of frontotemporal dementia: a cluster analysis study. Brain 2009; 132:2932-2946.

4. Neary D, Snowden JS, Northen B, Goulding P. Dementia of frontal lobe type. J Neurol Neurosurg Psychiatry 1988; 51:353-361.

5. Borroni B, Grassi M, Premi E, et al. Neuroanatomical correlates of behavioural phenotypes in behavioural variant of frontotemporal dementia. Behav Brain Res 2012;235: 124-129.

6. Ranasinghe KG, Rankin KP, Pressman PS, et al. Distinct subtypes of behavioral variant frontotemporal dementia based on patterns of network degeneration. JAMA Neurol 2016;73:1078-1088.

7. Franceschi M, Anchisi D, Pelati O, et al. Glucose metabolism and serotonin receptors in the frontotemporal lobe degeneration. Ann Neurol 2005;57:216-225.

8. Le Ber I, Guedj E, Gabelle A, et al. Demographic, neurological and behavioural characteristics and brain perfusion SPECT in frontal variant of frontotemporal dementia. Brain 2006;129:3051-3065.

9. Peters F, Perani D, Herholz K, et al. Orbitofrontal dysfunction related to both apathy and disinhibition in frontotemporal dementia. Dement Geriatr Cogn Disord 2006; 21:373-379.

10. Zamboni G, Huey ED, Krueger F, Nichelli PF, Grafman J. Apathy and disinhibition in frontotemporal dementia: insights into their neuronal correlates. Neurology 2008; 71:736-742.

11. Snowden JS, Bathgate D, Varma A, Blackshaw A, Gibbons ZC, Neary D. Distinct behavioural profiles in 
frontotemporal dementia and semantic dementia. J Neurol Neurosurg Psychiatry 2001;70:323-332.

12. Luzzi S, Fabi K, Pesallaccia M, Cafazzo V, Silvestrini M, Provinciali L. To clap or not to clap? Applause sign in apathetic and disinhibited/stereotypical behavioural variants of FTD. J Neurol Neurosurg Psychiatry 2014;85: 823-824.

13. O'Connor CM, Clemson L, Hornberger M, et al. Longitudinal change in everyday function and behavioral symptoms in frontotemporal dementia. Neurol Clin Pract 2016;6:419-428.

14. Josephs K, Whitwell JL, Weigand SD, et al. Predicting functional decline in behavioural variant frontotemporal dementia. Brain 2011;134:432-448.

15. Hsieh S, Schubert S, Hoon C, Mioshi E, Hodges JR. Validation of the Addenbrooke's Cognitive Examination III in frontotemporal dementia and Alzheimer's disease. Dement Geriatr Cogn Disord 2013;36:242-250.

16. Morris JC. The Clinical Dementia Rating (CDR): current version and scoring rules. Neurology 1993;43:2412-2414.

17. Wear HJ, Wedderburn CJ, Mioshi E, et al. The Cambridge Behavioural Inventory Revised. Dement Neuropsychol 2008;2:102-107.

18. Gelinas I, Gauthier L, McIntyre M, Gauthier S. Development of a functional measure for persons with Alzheimer's disease: the Disability Assessment for Dementia. Am J Occup Ther 1999;53:471-481.

19. Mioshi E, Dawson K, Mitchell J, Arnold R, Hodges JR. The Addenbrooke's Cognitive Examination Revised (ACE-R): a brief cognitive test battery for dementia screening. Int J Geriatr Psychiatry 2006;21:1078-1085.

20. Hsieh S, McGrory S, Leslie F, et al. The Mini-Addenbrooke's Cognitive Examination: a new assessment tool for dementia. Dement Geriatr Cogn Disord 2014;39:1-11.

21. Mooi E, Sarstedt M. Cluster Analysis. A Concise Guide to Market Research. Berlin: Springer; 2011:237-284.

22. Yim O, Ramdeen KT. Hierarchical cluster analysis: comparison of three linkage measures and application to psychological data. Quantitative Methods Psychol 2015;11: $8-21$.
23. Glickman ME, Rao SR, Schultz MR. False discovery rate control is a recommended alternative to Bonferroni-type adjustments in health studies. J Clin Epidemiol 2014;67: 850-857.

24. Lieberman MD, Cunningham WA. Type I and type II error concerns in fMRI research: re-balancing the scale. Soc Cogn Affect Neurosci 2009;4:423-428.

25. Dejesus-Hernandez M, Mackenzie IR, Boeve BF, et al. Expanded GGGGCC hexanucleotide repeat in noncoding region of C9ORF72 causes chromosome 9p-linked FTD and ALS. Neuron 2011;72:245-256.

26. Chow TW, Binns MA, Cummings JL, et al. Apathy symptom profile and behavioral associations in frontotemporal dementia vs dementia of Alzheimer type. Arch Neurol 2009;66:888-893.

27. Borroni B, Agosti C, Premi E, et al. The FTLD-modified Clinical Dementia Rating scale is a reliable tool for defining disease severity in frontotemporal lobar degeneration: evidence from a brain SPECT study. Eur J Neurol 2010; 17:703-707.

28. Robert P, Onyike CU, Leentjens AF, et al. Proposed diagnostic criteria for apathy in Alzheimer's disease and other neuropsychiatric disorders. Eur Psychiatry 2009; 24:98-104.

29. O'Connor CM, Clemson L, Brodaty H, Gitlin LN, Piguet $\mathrm{O}$, Mioshi E. Enhancing caregivers' understanding of dementia and tailoring activities in frontotemporal dementia: two case studies. Disabil Rehabil 2016;38:704-714.

30. de Vugt ME, Stevens F, Aalten P, Lousberg R, Jaspers N, Verhey FRJ. A prospective study of the effects of behavioral symptoms on the institutionalization of patients with dementia. Int Psychogeriatr 2005;17:577-589.

31. Diehl-Schmid J, Richard-Devantoy S, Grimmer T, Forstl $\mathrm{H}$, Jox R. Behavioral variant frontotemporal dementia: advanced disease stages and death: a step to palliative care. Int J Geriatr Psychiatry Epub 2016 Jul 4.

32. Massimo L, Powers JP, Evans LK, et al. Apathy in frontotemporal degeneration: neuroanatomical evidence of impaired goal-directed behavior. Front Hum Neurosci 2015;9:611.

\section{Apply Now for a BEBRF Research Grant}

The BEBRF is pleased to offer, to qualified researchers, funds of up to $\$ 100,000$ annually for research into new treatments, pathophysiology, and genetics of benign essential blepharospasm (BEB) and Meige Syndrome (cranial and oromandibular dystonia). Research into photophobia, dry eye and apraxia of eyelid opening, and their treatment, will also be considered for funding. Research proposals must relate specifically to BEB and Meige. Non-U.S. citizens working at institutions abroad are eligible to apply for a research grant.

Deadline to apply for a research grant is August 31, 2017. Grant guidelines can be obtained by email: bebrf@blepharospasm.org or downloaded from: www.blepharospasm.org 


\section{Neurology}

\section{Behavioral-variant frontotemporal dementia: Distinct phenotypes with unique functional profiles}

Claire M. O'Connor, Ramon Landin-Romero, Lindy Clemson, et al. Neurology 2017;89;570-577 Published Online before print July 12, 2017

DOI 10.1212/WNL.0000000000004215

This information is current as of July 12, 2017

\section{Updated Information \& Services \\ Supplementary Material}

\section{References}

Citations

Subspecialty Collections

Permissions \& Licensing

Reprints including high resolution figures, can be found at: http://n.neurology.org/content/89/6/570.full

Supplementary material can be found at: http://n.neurology.org/content/suppl/2017/07/12/WNL.0000000000004 215.DC1

This article cites 30 articles, 6 of which you can access for free at: http://n.neurology.org/content/89/6/570.full\#ref-list-1

This article has been cited by 2 HighWire-hosted articles: http://n.neurology.org/content/89/6/570.full\#\#otherarticles

This article, along with others on similar topics, appears in the following collection(s):

\section{Frontotemporal dementia}

http://n.neurology.org/cgi/collection/frontotemporal_dementia MRI

http://n.neurology.org/cgi/collection/mri

Information about reproducing this article in parts (figures,tables) or in its entirety can be found online at:

http://www.neurology.org/about/about_the_journal\#permissions

Information about ordering reprints can be found online:

http://n.neurology.org/subscribers/advertise

Neurology ${ }^{\circledR}$ is the official journal of the American Academy of Neurology. Published continuously since 1951, it is now a weekly with 48 issues per year. Copyright Copyright ( 2017 The Author(s). Published by Wolters Kluwer Health, Inc. on behalf of the American Academy of Neurology. All rights reserved. Print ISSN: 0028-3878. Online ISSN: 1526-632X.

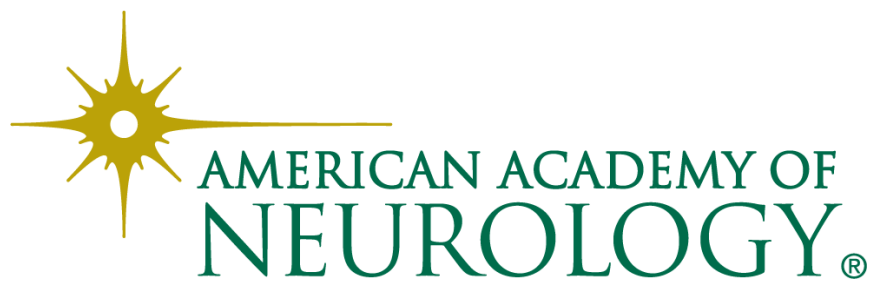

